\title{
Wing-Click Sounds of Heliconius cydno alithea (Nymphalidae: Heliconiinae) Butterflies
}

\author{
Mirian Medina Hay-Roe ${ }^{1}$ and Richard W. Mankin ${ }^{2,3}$
}

Accepted December 18, 2003; revised January 23, 2004

Field-collected Heliconius cydno Doubleday females were observed producing audible wing clicks during encounters between conspecifics in greenhouses in a large insectary during the day and at roosting time. Occasionally, these females also were observed producing sounds in aggressive encounters with females of a close relative, $\mathrm{H}$. erato $(L)$. However, the wing-clicks were not observed subsequently from first-generation adults born in the greenhouses. The sounds were produced in short trains of 3-10 wing-clicks at the rate of $\sim 10$ clicks/s. The individual clicks had a mean duration of $1.48 \mathrm{~ms}$ and a broad frequency spectrum, with a peak near $1075 \mathrm{~Hz}$. This peak lies near the 1200-Hz frequency of maximal sensitivity measured previously for auditory neurons of $\mathrm{H}$. erato. The production of these previously unreported sounds suggests that wing clicks may play a role in both intra-and interspecific communication among Heliconius species.

KEY WORDS: communication; sound; Heliconius cydno.

\section{INTRODUCTION}

Only a few butterfly species have been reported to produce or hear sounds. The most well-known examples are in the nymphaline butterfly genus, Hamadryas, which produce loud clicks (Yack et al., 2000, and references

${ }^{1}$ Department of Entomology and Nematology, University of Florida, Gainesville, Florida, 32611.

${ }^{2}$ USDA-ARS Center for Medical, Agricultural, and Veterinary Entomology, P. O. Box 14565, Gainesville, Florida, 32604. Fax: (352) 374-5781. e-mail: rmankin@gainesville.usda.ufl.edu.

${ }^{3}$ To whom correspondence should be addressed. 
therein). The behavioral function of wing clicks in Hamadryas has remained a question of considerable discussion since a proposal by Darwin (1874) that they may play a role in courtship. Others have suggested that the clicks startle predators or competitors (Young and Borkin, 1985) or that they are involved in male-male territoriality (Monge-Nájera et al., 1998).

In addition to Hamadryas, Heliconius spp. have been reported to hear and respond to clicks, but until now, there has been no evidence to indicate that they produce sounds (Yack et al., 2000; Swihart, 1967). When fieldcollected H. cydno alithea Doubleday from Ecuador were heard producing clicks in interactions with conspecifics, it provided an opportunity to record from Heliconius butterflies as they produced the clicks and then observe the behavioral responses to the sound.

Field-collected $H$. cydno produced clicks for about a month after their initial release into greenhouses in a large insectary in the Patterson Laboratories at the University of Texas, Austin. The first-generation adults born in the greenhouses did not produce any perceptible sounds. Sounds were also produced during interspecific interactions with Heliconius erato phyllis; however, none of these sounds was recorded because the encounters were brief.

\section{METHODS}

To record sounds, a Panasonic microcassette recorder (Matsushita, Inc., Osaka, Japan) was held near the butterflies in the greenhouse. Sounds were recorded near midday, when males and females were actively flying and feeding, and late in the afternoon, when they began to roost.

For acoustic analysis, the signals in seven separate wing-click trains were band-pass filtered between 30 and $6000 \mathrm{~Hz}$, digitized at $25 \mathrm{kHz}$, and analyzed using a custom-written signal acquisition and analysis program (DAVIS, [see Mankin et al., 2000a, b, for details]). Relative differences in signal amplitude and confirmation by audio playback of signals were used to identify signal peaks as wing clicks (see examples in Fig. 1). A subroutine in the DAVIS program placed the peak of each click (e.g., Fig. 1b) at the center of a 512point ( $20.48 \mathrm{~ms}$ ) window, applied a Hamming filter, and calculated a discrete Fourier-transform power spectrum (Mankin et al., 2000a). Spectral averages of multiple clicks were constructed by averaging spectrum levels of all clicks at each discrete reference frequency (e.g., Embree and Kimble, 1991).

\section{RESULTS AND DISCUSSION}

The clearest recordings were obtained at roosting time, about 1800 1830 on April 21, 1995. The clicks occurred when the flying and roosting 


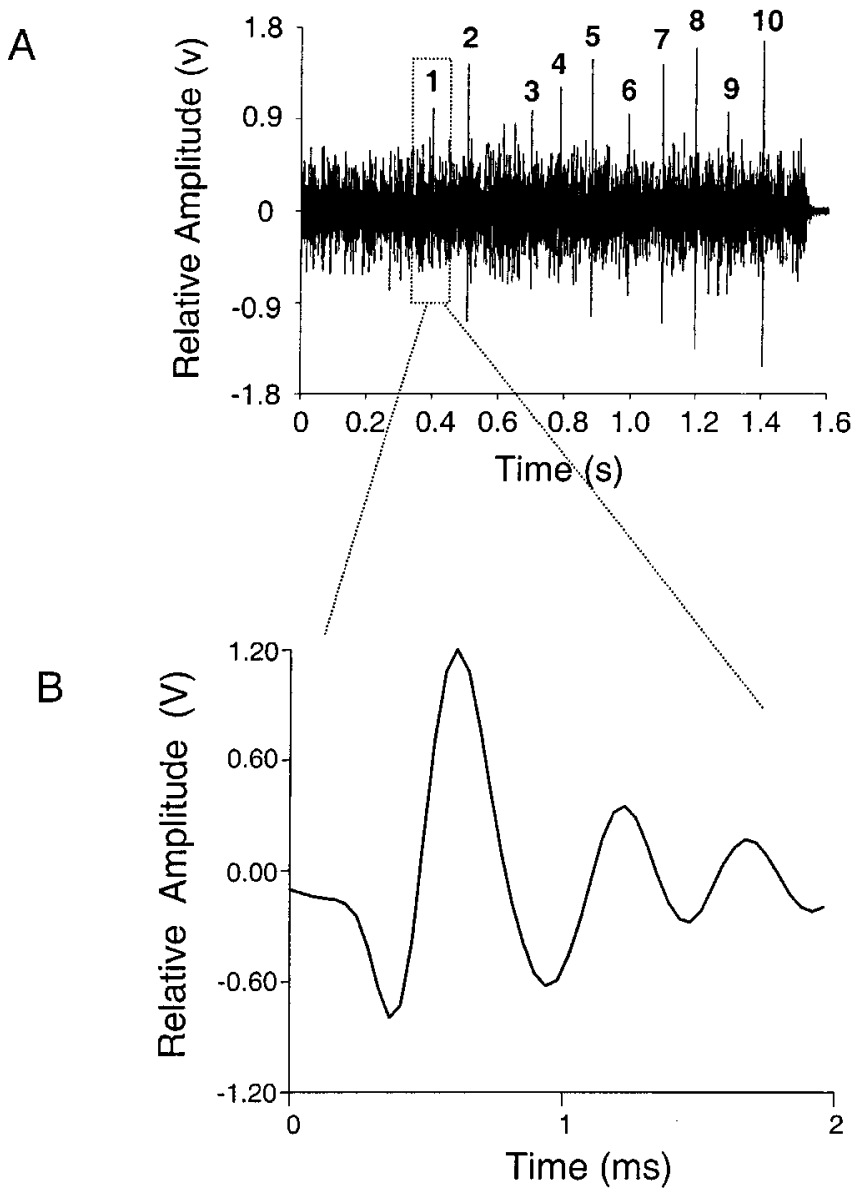

Fig. 1. (A) Oscillogram of a train of 10 wing-clicks generated by a hovering female $H$. cydno. The horizontal axis shows the time in seconds. The vertical axis shows the microphone signal on a relative scale. (B) Expanded view of first click (No. 1) on a 1-ms time scale.

individuals came very close to each other, almost touching each other's heads and wings. Similar sounds were also recorded earlier that day when a male and female encountered each other. The individual perching in the branch or stem of the roosting site responded to the sound-producing individual by beating its wings in a slow, rhythmic manner.

Although we did not record interspecific sounds, the first author heard clear sounds produced by $H$. cydno in encounters with $H$. erato phyllis. During these encounters, the field-collected $H$. cydno behaved aggressively, 
chasing the $H$. erato away by flying at high speed, thereby gaining access to Psiguria flowers upon which they feed.

The wing clicks produced by flying $H$. cydno females were short, broadband pulses. In the longest recording from the encounter between a flying and a roosting female (20 clicks over a 5.5-s period), the clicks had a mean duration of $1.48 \pm 0.14 \mathrm{MS}$ (SE) and a peak energy near $\sim 1075 \mathrm{~Hz}$ (see mean spectrum between 0 and $3 \mathrm{kHz}$ in Fig. 2). The mean relative signal level gradually decreased to $-42 \mathrm{~dB}$ between 3 and $6 \mathrm{kHz}$. Six of the 20 clicks had two components, possibly an indication that each wing contributed separate sounds during the wingbeat cycle (e.g., Yack et al., 2000). For these six clicks, the mean time between components was $0.77 \pm 0.11 \mathrm{~ms}$. Because of this slight separation between components, the mean durations of the double-component clicks were longer $(2.27 \mathrm{~ms})$ than the mean durations of the 14 clicks with only one apparent component $(1.14 \mathrm{~ms})(t=8.05, \mathrm{df}=18$, $P<0.01)$.

The recorded sounds occurred in short trains of 3-10 clicks. The time between the peaks of clicks in four trains that contained four or more clicks each was measured as interclick interval (ICI), and the click frequency was calculated as 1/ICI (Fig. 3). The mean click frequency of the trains in Fig. 3 was $10.9 \pm 0.7 \mathrm{~Hz}$.

The clicks recorded from $H$. cydno were weaker and lower in frequency than those that have been recorded from Hamadryas feronia (Frushtorfer),

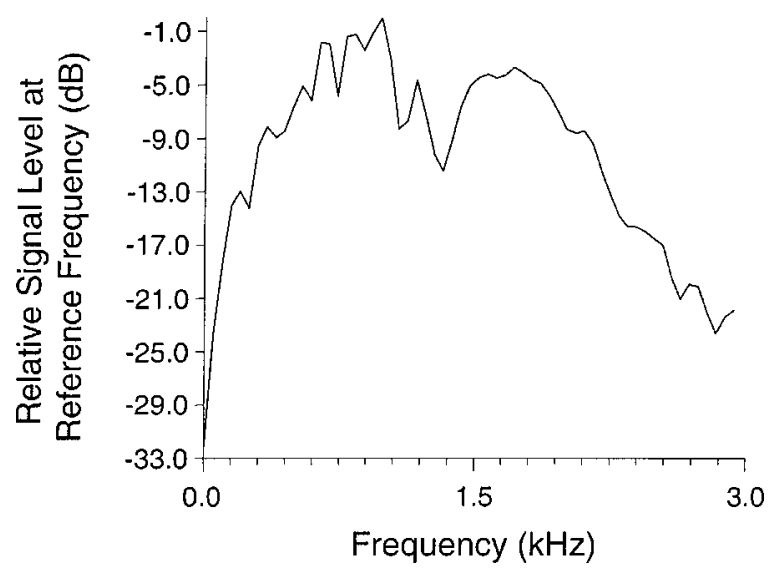

Fig. 2. Average spectrum of a train of 10 clicks produced by a hovering female H. cydno. The horizontal axis shows the frequency in kilohertz. The vertical axis indicates the relative signal level (decibels relative to maximum signal decibels) at the reference frequency. 


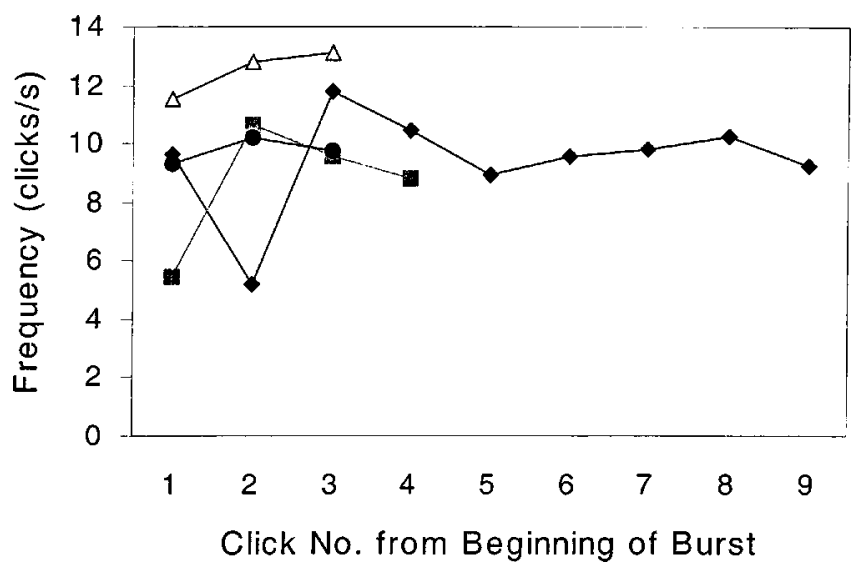

Fig. 3. Temporal pattern of $H$. $c y d n o$ wing-clicks in four separate trains recorded from an encounter between two females. The vertical axis indicates the click frequency (1/ICI) in clicks per seconds. The horizontal axis indicates the number of clicks from the beginning of each train.

the butterfly whose sound production and hearing have been studied in the greatest detail (Yack et al., 2000; Swihart, 1967). Yack et al. (2000) found that $H$. feronia males produced pairs of loud (80- to $100-\mathrm{dB}$ sound pressure level [SPL] at $10 \mathrm{~cm}$ ) clicks with durations of $0.42 \mathrm{~ms}$ and peak energies between 13 and $15 \mathrm{kHz}$. The microcassette recording system is not ideal for calibrated, high-frequency acoustic measurements, so we did not perform analyses at frequencies $>6 \mathrm{kHz}$. However, an approximate calibration of the signal between 0 and $6 \mathrm{kHz}$ (see Mankin et al., 1996) indicated that the Heliconius wing clicks were weak sounds $(<40-\mathrm{dB}$ SPL at $10 \mathrm{~cm})$. The recorded signals had a minimal energy of between 3 and $6 \mathrm{kHz}$. Subjectively, the clicks played back from recordings sounded very similar to clicks heard directly; consequently, we do not consider it likely that the clicks contained significant energy at frequencies between $6 \mathrm{kHz}$ and the upper frequencies of human hearing. In contrast, the $80-\mathrm{dB}$ clicks by $H$. feronia males usually had greater energy between 3 and $6 \mathrm{kHz}$ than between 0 and $3 \mathrm{kHz}$ (Yack et al., 2000) and, thus, would seem much louder and higher in frequency to a human observer. The differences in loudness and frequency suggest that the clicks were produced by different mechanisms in the two butterfly species. The precise mechanism remains to be determined in further studies, but it has been proposed that the sound is produced by a percussive mechanism in Hamadryas (Otero, 1990; Monge-Nájera et al., 1998).

One of the observed behaviors associated with sound production was that the signaling butterfly often flew very close to the other butterfly, almost 
touching its head and wings. This behavior is perhaps associated with the presence of a secondary sound receptor organ found in the head. Swihart (1967) found that the greatest number of auditory receptors appeared in the optic lobes, which are particularly large in Heliconius (Gilbert, 1975, and references therein). Swihart also determined that the sounds were coming from metathoracic wing nerve IIIN1c. The sensitivity of IIIN1c was greatest at $1.2 \mathrm{kHz}$, near the peak frequency of the clicks produced by H. cydno. However, because sound production by Heliconius had not been observed prior to the current study, there was some doubt as to whether hearing plays a functional role in interspecific communication in this genus.

The observations of field-collected $H$. cydno producing clicks in encounters among conspecifics and members of different species are consistent with a hypothesis that hearing plays a functional role in intra- and interspecific communication in Heliconius. Furthermore, because sounds from one species potentially could be perceived by others (e.g., H. erato is anatomically equipped to detect sounds produced by cydno), these clicking sounds could serve as means of communication between members of multiple species in a butterfly community.

It is unlikely that sound production by adult Heliconius functions as a defensive mechanism as in Arctiidae and Noctuidae moths (e.g., Roeder, 1962; Blest, 1963). Heliconius butterflies present another type of defensive mechanism against predation, which is advertised through their warming coloration (Brower, 1989; Chai and Srygley, 1990). Future studies may clarify whether sound production is a widespread phenomenon within Heliconius and whether members of different species (e.g., within and between mimicry rings) can use sound to communicate with one another.

Finally, we do not have a clear explanation for why the butterflies ceased producing sounds within a month after they were released in the greenhouses, or why the first generation of adults born in the greenhouses did not produce sounds. However, sound production may be inhibited by loud noises produced by fans and other electrical equipment (see, e.g., Römer et al., 1989). Another type of communication such as semiochemical communication may be more effective under greenhouse conditions.

\section{ACKNOWLEDGMENTS}

We thank Everett Foreman and Betty Weaver (Center for Medical, Agricultural, and Veterinary Entomology) for signal and graphical analysis, Carla Penz, Frank Slansky, and Tom Emmel for comments on early drafts of the manuscript, and Durell Kapan for bringing wild H. cydno alithea from Ecuador. Part of this research was performed under the support of L. Gilbert's NSF grant. 


\section{REFERENCES}

Blest, A. D., Collett, T. S., and Pye, J. D. (1963). The generation of ultrasonic signals by a New World arctiid moth. Proc. Roy Soc. Lond. B 158: 196-207.

Brower, L. P. (1989). Chemical defenses in butterflies. In Vane-Wright, R. I., and Ackery, P. R. (ed.), The Biology of Butterflies, Princeton University Press, Princeton, NJ.

Chai, P., and Srygley, R. B. (1990) Predation and the flight, morphology, and temperature of neotropical rainforest butterflies. Am. Nat. 135: 748-765.

Darwin, C. R. (1874). The Descent of Man, Prometheus Books, New York.

Embree, P. M., and Kimble, B. (1991). C Language Algorithms for Digital Signal Processing, Prentice-Hall, Englewood Cliffs, NJ.

Gilbert, L. E. (1975). Ecological consequences of a coevolved mutualism between butterflies and plants. In Gilbert, L. E., and Raven, P. R. (eds.), Coevolution of Animals and Plants, University of Texas Press, Austin.

Mankin, R. W., Shuman, D., and Coffelt, J. A. (1996). Noise shielding of acoustic devices for insect detection. J. Econ. Entomol. 89: 1301-1308.

Mankin, R. W., Brandhorst-Hubbard, J., Flanders, K. L., Zhang, M., Crocker, R. L., Lapointe, S. L., McCoy, C. W., Fisher, J. R., and Weaver, D. K. (2000a). Eavesdropping on insects hidden in soil and interior structures of plants. J. Econ. Entomol. 93: 1173-1182.

Mankin, R. W., Petersson, E., Epsky, N. D., Heath, R. R., and Sivinski, J. (2000b). Exposure to male pheromones enhances Anastrepha suspensa (Diptera: Tephritidae) female response to male calling song. Fla. Entomol. 83: 411-421.

Monge-Nájera, J. Hernández, F., González, M. I., Soley, J. Araya, J., and Zolla, S. (1998). Spatial distribution, territoriality, and sound production by tropical cryptic butterflies (Hamadryas, Lepidoptera: Nymphalidae): Implications for the 'Industrial Melanism' debate. Rev. Biol. Trop. 48: 297-329.

Otero, L. D. (1990). The stridulatory organ in Hamadryas (Nymphalidae): Preliminary observations. J. Lepidopt. Soc. 44: 285-288.

Roeder, K. D. (1962). The behavior of free-flying moths in the presence of artificial ultrasonic pulses. Anim. Behav. 10: 300-304.

Römer, H., Bailey, W., and Dadour, I. (1989). Insect hearing in the field. III. Masking by noise. J. Comp. Physiol. A. 164: 609-620.

Swihart, S. L. (1967). Hearing in butterflies (Nymphalidae: Heliconius, Ageronia). J. Insect Physiol. 13: 467-476.

Yack, J. E., Otero, L. D., Dawson, J. W., Surlykke, A., and Fullard, J. H. (2000). Sound production and hearing in the blue cracker butterfly Hamadryas feronia (Lepidoptera, Nymphalidae) from Venezuela. J. Exp. Biol. 203: 3689-3702.

Young, A. M., and Borkin, S. S. (1985). Natural history notes for some Hamadryas butterflies (Nymphalidae: Nymphalinae: Aegeronini) in northwestern Costa Rica during the tropical dry season. J. Lepidopt. Soc. 39: 229-235. 